\title{
Efecto de la permeabilidad en la regeneración del nervio ciático entubado de rata
}

\begin{abstract}
Hernán Hurtado
\section{Resumen}

Se seccionaron nervios ciáticos de rata adulta y se introdujeron en cámaras de regeneración dejando un espacio de $10 \mathrm{~mm}$ entre las extremidades del nervio. En las paredes de estas cámaras, se practicaron 2 o 9 agujeros de $4 \mathrm{~mm}^{2}$. En otro grupo de animales, no se practicó ningún agujero en las cámaras. Una, dos y cuatro semanas después de la cirugía, se perfundieron los animales y se evaluó la histología de los regenerados. Adicionalmente, a las cuatro semanas se contó el número de fibras mielínicas y se midió el área de los regenerados. Se observa una aceleración del proceso de regeneración que depende del número de agujeros practicados. El número de fibras mielínicas y el área del regenerado también se incrementan con el número de agujeros.
\end{abstract}

\section{Summary}

Sectioned rat sciatic nerves were placed in regeneration chambers, leaving a gap of 10 $\mathrm{mm}$ between the proximal and distal stumps. The walls of these chambers carried none, two or nine $4 \mathrm{~mm}^{2}$ holes. One, two or four weeks after the surgery, animals were perfused and regenerates' histology was evaluated. In addition, in the 4 week group, the number of myelinated fibers and the surface of the regenerates were evaluated at mid-tube level. An acceleration of the regenerative process was observed, depending on the number of holes. The number of myelinated fibers and the size of the regenerates also increase proportionally with the number of holes in the tubes.

A pesar de los avances introducidos en las técnicas microquirúrgicas, la sección de un tronco nervioso todavía conlleva problemas clínicos, sociales y económicos para los individuos afectados. Además, las condiciones biológicas que pueden llevar a una mejoría en la regeneración de nervios periféricos y, especialmente, a recobrar la función perdida, son aún poco claros. El modelo de la entubación se ha convertido en una herramienta experimental muy útil para el estudio de los fenómenos básicos subyacentes en la regeneración del nervio periférico (1-3). Las guías tubulares que se han utilizado son hechas de diversos materiales y permeabilidades: sintéticas impermeables, semipermeables o totalmente permeables; biodegradables o de origen biológico (4-9). En la rata, los tubos de silicona impermeable permiten la regeneración de un mayor número de fibras mielínicas que los tubos acrílicos semipermeables (10). Sin embargo, se ha demostrado que en tubos totalmente permeables, el proceso de regeneración ocurre más rápido que en los demás (11).

En este trabajo se demuestra que el número de fibras mielínicas que se encuentran a nivel medio de una cámara de regeneración, depende del número de agujeros macroscópicos que se le practiquen a dicha cámara. También se demuestra un incremento en la velocidad de

Coordinador de investigaciones, Laboratorio de Neurociencias, Instituto Nacional de Salud, Santa Fe de Bogotá. 
regeneración que se detecta en las etapas iniciales del proceso, a nivel de la colonización de la matriz de fibrina-fibronectina por células de morfología fibroblástica.

\section{Materiales y métodos}

Los experimentos se llevaron a cabo en 30 ratas Wistar, machos de 2 a 5 meses de edad. Los animales se anestesiaron con Hypnorm (Duphar, $0,15 \mathrm{~mL} / 100 \mathrm{~g}$ ) administrado intramuscularmente. La piel y los músculos de la pata posterior derecha se cortaron con tijeras, y el nervio ciático se movilizó y seccionó a nivel medio del muslo. La entubación se llevó a cabo en dos etapas: en la primera, las extremidades proximales y distales del nervio seccionado se insertaron dentro de tubos de silicona de $2 \mathrm{~mm}$ de longitud, $1 \mathrm{~mm}$ de diámetro interno y $3 \mathrm{~mm}$ de diámetro externo. Estos tubos se insertaron luego dentro de tubos de silicona de $14 \mathrm{~mm}$ de longitud, $3 \mathrm{~mm}$ de diámetro interno y $5 \mathrm{~mm}$ de diámetro externo. Los muñones nerviosos se mantuvieron en su sitio por medio de suturas 90 , dejando un espacio de $10 \mathrm{~mm}$ entre ellos. Después de la cirugía, los músculos se cerraron con hilo 5-0 y la piel con ganchos Michel.

Después de un tiempo de supervivencia de 1 $(n=9), 2(n=9)$ o $4(n=12)$ semanas, los animales fueron anestesiados y perfundidos intraaórticamente con una solución de lavado (cloruro de sodio $0,8 \%$, glucosa $0,1 \%$ y clorhidrato de procaína $0,1 \%$ ) seguida por una solución fijadora (formaldehído $2 \%$ y glutaraldehído $2,5 \%$ ) en amortiguador fosfato a $\mathrm{pH}$ 7,4 (12). Las cámaras de silicona se retiraron y cortaron por la mitad. El tejido regenerado se sumergió en el mismo fijador durante 1 hora; se postfijó con tetraóxido de osmio $2 \%$ durante 1 hora; se deshidrató en concentraciones crecientes de alcohol y se embebió en Epon. Después de la polimerización, se obtuvieron cortes semifinos (1 $\mu \mathrm{m}$ de espesor) que se tiñeron con azul de toluidina. Para las observaciones en microscopio electrónico, se obtuvieron cortes ultrafinos $(90-100 \mathrm{~nm}$ de espesor) que se contrastaron con acetato de uranilo y citrato de plomo con el fin de establecer la presencia de fibras amielínicas y para estudiar la ultraestructura de los nervios regenerados.
Se utilizaron los siguientes grupos experimentales:

1- Tubos impermeables, tiempos de supervivencia de $1(n=3), 2(n=3) \circ 4(n=4)$ semanas.

2- Tubos con 2 agujeros de $4 \mathrm{~mm}^{2}$; de resto, semejante al grupo 1 .

3- Tubos con 9 agujeros; de resto, semejante al grupo 2.

En todos los grupos se estudió la histología del regenerado a nivel medio del tubo. Para los animales con tiempos de supervivencia de 1 y 2 semanas, sólo se hizo un estudio descriptivo de la histología de los regenerados. Para los animales con 4 semanas de supervivencia, se cuantificaron los números de fibras mielínicas y el área de los regenerados a nivel medio del tubo. Estas cuantificaciones se llevaron a cabo en cortes semifinos usando un Visopan (Reichert) a un aumento final de $1.200 \mathrm{X}$. La superficie $\left(\mathrm{mm}^{2}\right)$ de las mismas secciones se obtuvo utilizando un planímetro (Kontron). Los datos se analizaron utilizando el análisis de varianza de una vía, seguido de la prueba de Neuman Keuls de comparación múltiple de medias (13).

\section{Resultadios}

\section{Evaluación histológica}

Una semana de supervivencia: en todos los casos, se extiende una matriz fibrilar entre las dos extremidades del nervio seccionado. Esta estructura es similar a otras ya descritas en la literatura utilizando este sistema experimental $(6,8,9)$. La matriz presenta un aspecto difuso y contiene esencialmente células sanguíneas y fibrina. No se observan células de Schwann, ni axones, ni vasos sanguíneos. Sin embargo, sí se presenta una diferencia entre las estructuras regeneradas en tubos impermeables o con 2 agujeros (figura 1A) y aquéllas que lo hacen en tubos con 9 agujeros (figura $1 \mathrm{~B}$ ). Estas últimas son más compactas y se detectan células con morfología fibroblástica, cosa que no ocurre en los otros grupos.

Dos semanas de supervivencia: se observa una estructura bien organizada en todos los casos. 
Su madurez depende del número de agujeros practicados en los tubos. En las cámaras impermeables, las células de Schwann colonizan el regenerado y se distribuyen formando pequeñas unidades de regeneración (figura 2). A pesar de esto, no se detectan fibras mielínicas en microscopía de luz. Otros componentes celulares del regenerado son los fibroblastos y los vasos sanguíneos. En los tubos con 2 agujeros, los compartimentos están más desarrollados y presentan unidades de regeneración evidentes. Los regenerados dentro de los tubos con 9 agujeros son muy diferentes de los otros; son de mayor tamaño y están rodeados de una importante cantidad de tejido conectivo laxo. Dentro del regenerado, se observan unidades de regeneración de mayor tamaño con fibras que probablemente corresponden a fibras promielínicas (figura 3 ).

Cuatro semanas de supervivencia: para los tres grupos experimentales, la organización histológica básica es similar a otras descripciones ya publicadas $(6,9)$; en términos generales, se observa un puente circular de tejido conectivo, altamente vascularizado y colonizado por células de Schwann y fibras nerviosas mielínicas (figura 4A). Sin embargo, nuevamente se observa una diferencia importante entre los tubos con 9 agujeros y los otros. Se trata del extraordinario desarrollo de la capa de tejido conectivo externo que se presenta en el primer caso, en el cual se observan minifascículos (figura 4B).

\section{Análisis cuantitativo}

Una característica muy interesante que se observa a partir de estos experimentos es el incremento en el tamaño del regenerado y en el número de fibras mielínicas, en función del número de agujeros practicados en las paredes de los tubos. El cuadro 1 muestra el total de fibras mielínicas en regenerados de 4 semanas, a nivel medio del tubo, lo mismo que el área de estos regenerados. Es claro que los dos parámetros son proporcionales al número de agujeros.

El análisis estadístico revela que existe un efecto significativo del número de agujeros sobre
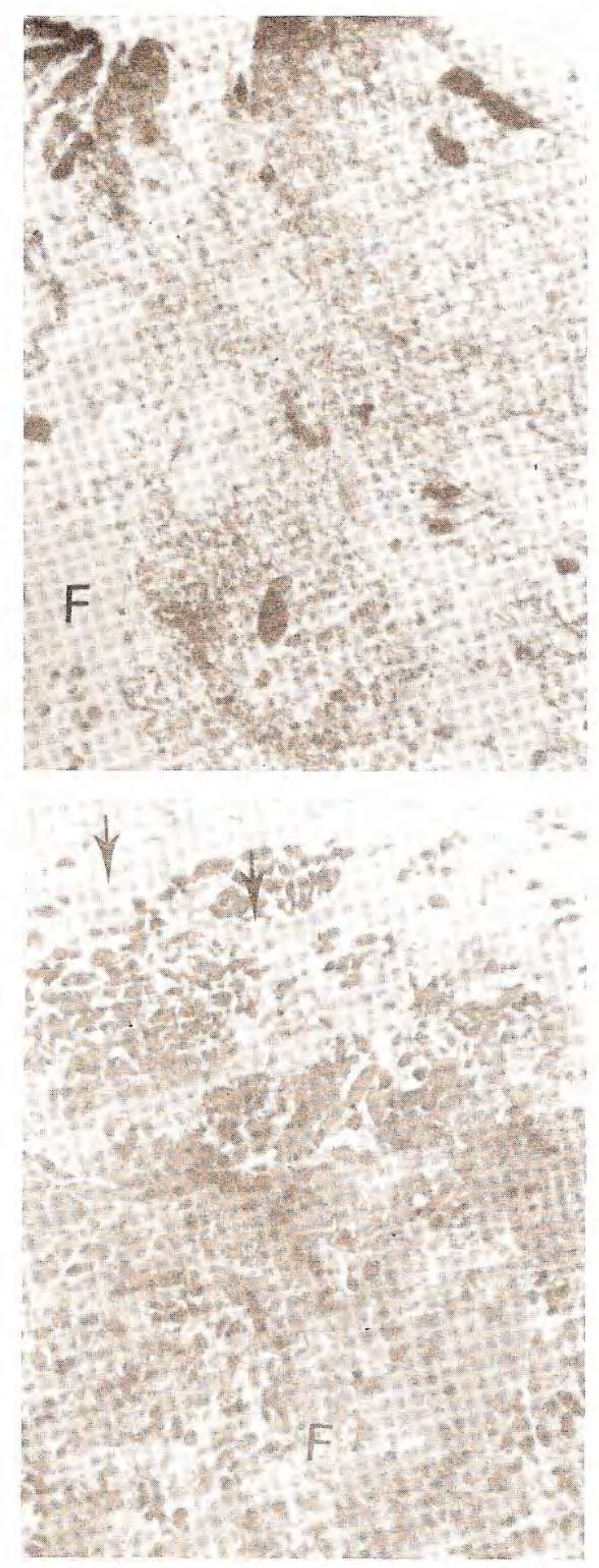

Figura 1. Una semana de regeneración. F: matriz de fibrina; flecha: célula fibroblástica $(800 \mathrm{X})$. A. Cámara impermeable. B. Cámara con 9 agujeros. 


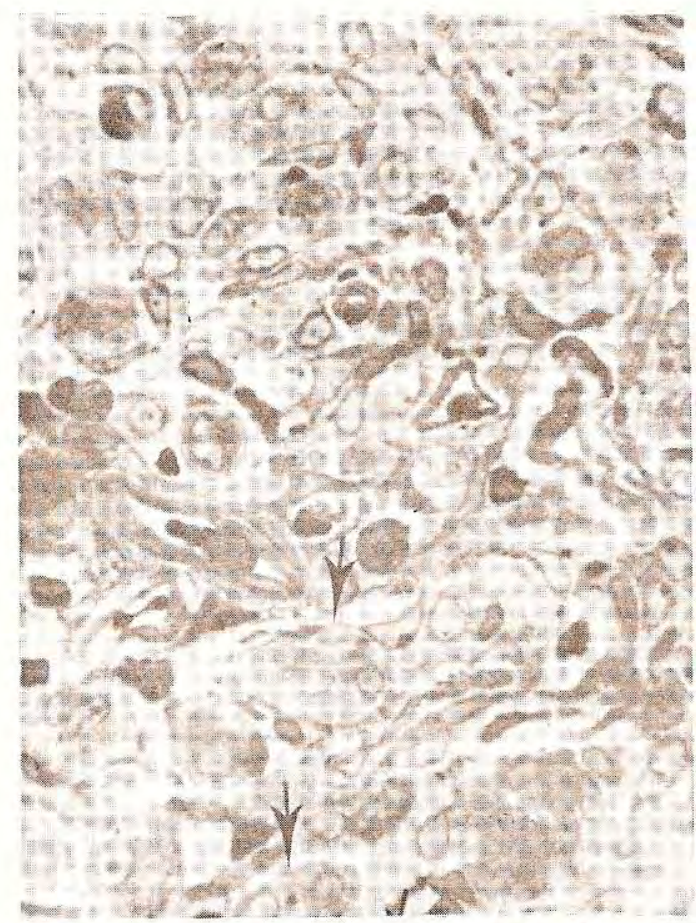

Figura 2. Dos semanas de regeneración: cámara impermeable. Flecha: unidades de regeneración $(900 \mathrm{X})$.

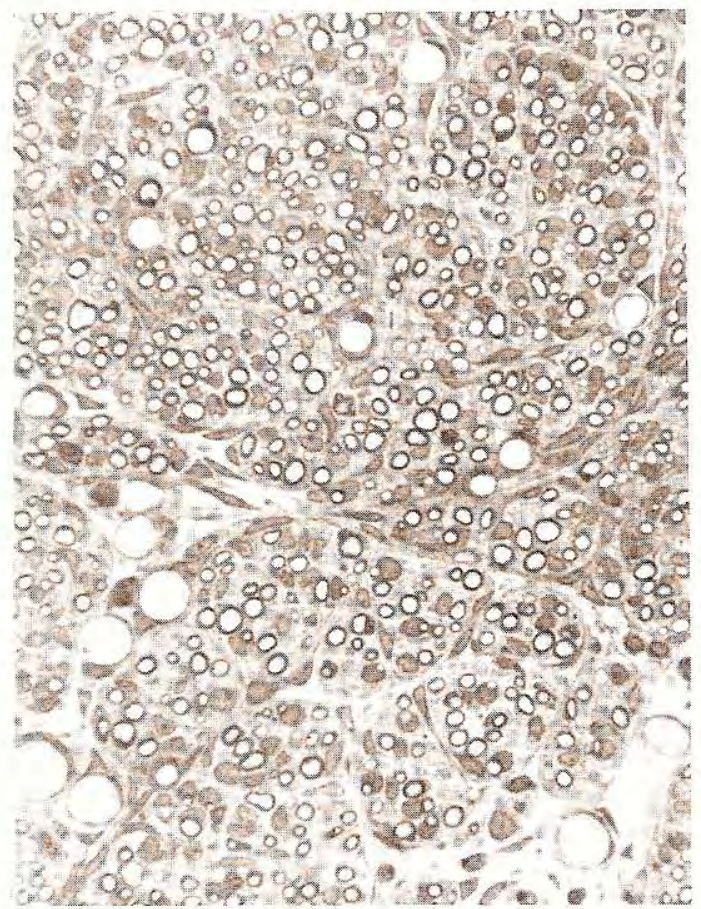

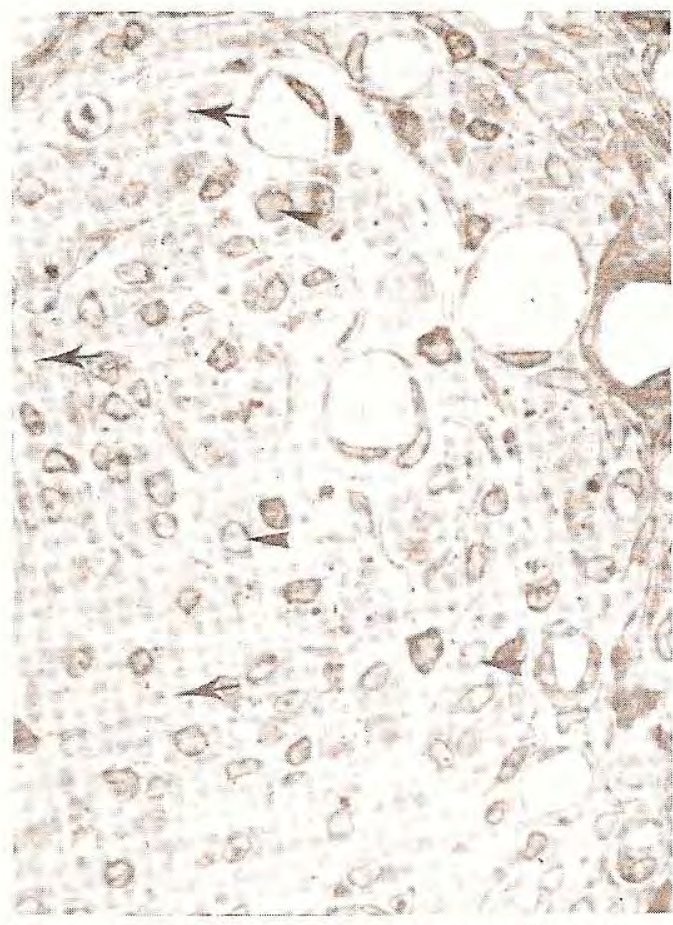

Figura 3. Dos semanas de regeneración: camara con 9 agujeros. Flecha: probables fibras promielínicas; cabeza de flecha: células de Schwann $(900 \mathrm{X})$.

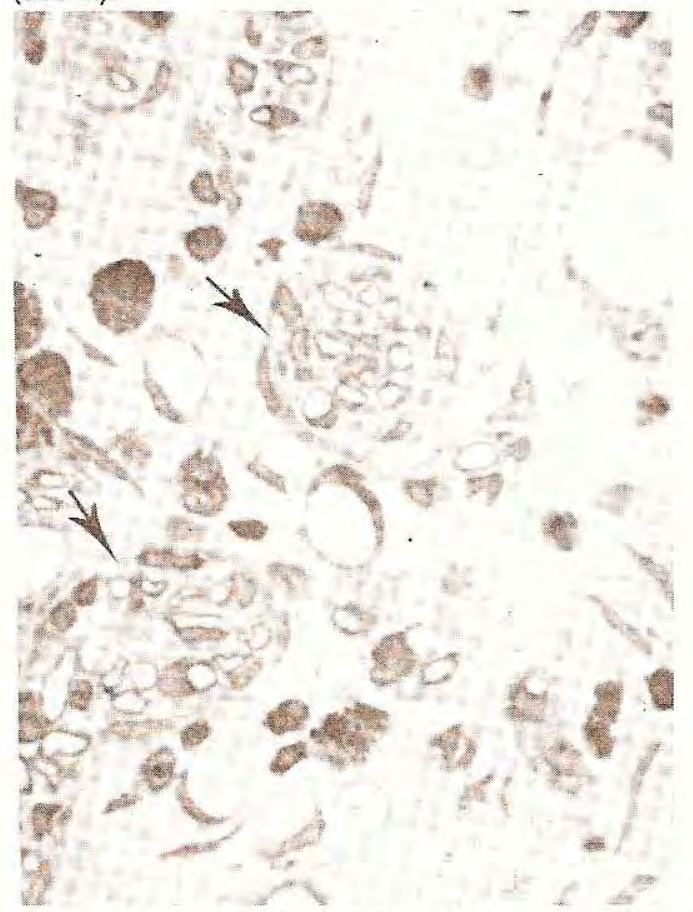

Figura 4. Cuatro semanas de regeneración: cámara con 9 agujeros. Flecha: minifascículos. A. Numerosas fibras mielínicas están presentes en el regenerado $(900 \mathrm{X})$. B. Minifascículos con fibras mielínicas inmersas en el tejido 
Cuadro 1. Nervio ciático entubado de rata: cuatro semanas de regeneración.

\begin{tabular}{lccc}
\hline $\begin{array}{l}\text { Número de } \\
\text { agujeros }\end{array}$ & 0 & 2 & 9 \\
\hline $\begin{array}{l}\text { Número de 203 } \\
\text { fibras }(X \pm D S)\end{array}$ & $2.112 \pm 2.117$ & $3.896 \pm 992$ \\
$\begin{array}{l}\text { Area del } 20,11 \pm 22,71 \\
\text { regenerado } \\
\left(\mu \mathrm{m}^{2}\right)(X \pm D S)\end{array}$ & $85,06 \pm 73,145$ & $31,99 \pm 174,12$ \\
\hline
\end{tabular}

los dos parámetros mencionados $(p<0,01$ y $p<0,05$ para el número de fibras y el área del regenerado, respectivamente). La prueba de Newman-Keuls revela una diferencia significativa en el número de fibras, entre los tubos impermeables y los de 9 agujeros.

Las otras comparaciones no muestran diferencias significativas. Por otro lado, en esta. misma tabla se observa que la superficie del regenerado en tubos de 9 agujeros es mayor que con los otros dos tipos de tubo $(p<0,01)$.

\section{Discusión}

La entubación del nervio ciático con tubos impermeables o totalmente permeables (con agujeros macroscópicos) demuestra que ios últimos permiten una más rápida regeneración en términos del área del regenerado y del número de fibras mielínicas. Estos resultados confirman y extienden las observaciones de Jenq y Coggeshall $(14,15)$. Estos autores demostraron que los tubos con 2 agujeros permiten una regeneración más rápida y un incremento en el número de fibras mielínicas en comparación con los tubos impermeables. Sin embargo, ellos analizaron solamente las etapas finales del proceso de regeneración en el sistema de cámara.

Como se describe en nuestro trabajo, las etapas iniciales del proceso parecen ser críticas para la formación de un nuevo nervio. Estos resultados demuestran que las fases iniciales de la regeneración son más rápidas en las cámaras totalmente permeables. No podemos descartar un efecto directo sobre las etapas más avanzadas (directamente sobre los axones). Sin embargo, la demostración de que, en presencia de varios agujeros, la colonización de la matriz por los fibroblastos ocurre más rápidamente que en ausencia de agujeros, nos lleva a pensar que muy probablemente lo que se está estimulando son las etapas iniciales de la regeneración.

Es necesario explicar la colonización acelerada de la matriz por células de morfología fibrobiástica. Ya se ha planteado que, en estas etapas iniciales, la regeneración se asemeja a un proceso de cicatrización (16). Se cree que dentro de los tubos (aún de los agujereados) se acumulan substancias activadoras del crecimiento y quimiotácticas, En los tubos impermeables, el papel de estas sustancias podría ser el de incrementar la población de fibroblastos a partir de las extremidades del nervio seccionado (17-24). En el caso de los tubos agujereados, existe una fuente extra de fibroblastos. Esta fuente es la vaina mesotelial que envuelve los tubos. Un puente celular se forma entre esta vaina y el regenerado. El efecto tan marcado del número de agujeros también es compatible con la hipótesis de la fuente externa de fibroblastos. Otros autores han demostrado que si se tapan los agujeros de tubos totalmente permeables con membranas de poros pequeños $(0,22 \mu \mathrm{m})$, la regeneración se lleva a cabo a una velocidad similar a la que existe en tubos impermeables (14). Estas observaciones también demuestran el papel tan importante de las células externas. Se puede suponer que la formación del puente de tejido conectivo sigue las mismas reglas que la reacción de cicatrización $(25,26)$ : establecimiento de una matriz de fibrina-fibronectinaácido hialurónico y su subsecuente colonización por los fibroblastos. Es importante aclarar que la etapa de formación de la matriz no ha sido abordada en este trabajo. Sin embargo, sí hemos estudiado la segunda etapa, es decir, la colonización de esta matriz por los fibroblastos. Los mecanismos que están implicados en la formación más rápida de esta matriz pueden ser similares a los sugeridos por Williams y Varon (27) para explicar la regeneración en cámaras llenas de solución salina. Las materias primas (fibrina y fibronectina) probablemente se diluyen y su difusión se incrementa. Además, la presencia de agujeros permite la entrada de 
exudado de la lesión (como resultado de la cirugía) hacia el interior de la cámara. Como ya ha sido descrito, los patrones electroforéticos del líquido que se acumula en las cámaras de regeneración y del líquido que se acumula en los tubos abiertos (00) son muy similares (16). Ya que la fuente más probable del líquido en tubos 00 es el exudado poslesional (28), es razonable suponer que éste contribuye a la formación del líquido de las cámaras de regeneración y a la matriz, en el caso de los tubos agujereados.

Es importante enfatizar que el aceleramiento de la regeneración (en términos de número de fibras mielínicas y área del regenerado) que se observa utilizando tubos agujereados, no necesariamente implica una mejor recuperación de la función. Aunque este trabajo no se diseñó para estudiar este último fenómeno, algunas de las observaciones sí son relevantes a ese respecto. Se sabe que la mala orientación de los axones en regeneración en el espacio que queda entre los muñones del nervio seccionado es una causa importante de fallas en reparaciones clínicas de nervios periféricos (1). La perturbación de los patrones intraneurales normales lleva a que los axones en crecimiento colonizen bandas de Büngner erradas.

En los tubos con 9 agujeros, hemos descrito la presencia de pequeñas unidades de regeneración dispersas dentro del regenerado, incluso en el epineurio recién formado. Pensamos que este patrón difuso hace difícil una reinervación correcta de los blancos. La entrada masiva de fibroblastos probablemente altera la topografía intraneural. Cada agujero es una fuente de perturbación del patrón normal. Por tanto, más agujeros pueden llevar a una mayor desorganización y a una mala inervación de los órganos blanco. Es necesario recalcar que los agujeros que se utilizaron en este trabajo son bastante grandes $\left(4 \mathrm{~mm}^{2}\right)$. Probablemente, la utilización de mallas de tamaño de poro menor podrían resultar en una entrada más equilibrada de fibroblastos al interior de los tubos. Actualmente, estamos realizando experimentos con el fin de examinar esta hipótesis.

\section{Referencias}

1. Lundborg G. Nerve injury and repair. Edinburgh: Churchill Livingstone; 1988.

2. Fields RD, Le Beau JM, Longo FM, Ellisman MH. Nerve regeneration through artificial tubular implants. Prog Neurobiol 1989;33:87-134.

3. Horowitz SH. Therapeutic strategies in promoting peripheral nerve regeneration. Muscle \& Nerve 1989; 12:314-22.

4. Lundborg G, Hansson HA. Regeneration of peripheral nerve through a preformed tissue space. Preliminary observations on the organization of regenerating nerve fibers and perineurium. Brain Res 1979 178:573-6.

5. Chiu DTW, Janecka I, Krizek TJ, Wolff M, Lovelace RE. Autogenous vein graft as a conduit for nerve regeneration. Surgery 1982;91:226-33.

6. Williams LR, Longo FM, Powell HC, Lundborg G, Varon S. Spatial-temporal progress of peripheral nerve regeneration within a silicone chamber: parameters for a bioassay. J Comp Neurol 1983;218:460-70.

7. Seckel BR, Chiu TH, Nyilas E, Sidman RL. Nerve regeneration through synthetic biodegradable nerve guides: regulation by the target organ. Plast Reconstr Surg 1984;74:173-81.

8. Uzman BG, Villegas GM. Mouse sciatic nerve regeneration through semipermeable tubes: a quantitative model. J Neurosci Res 1983;9:325-38.

9. Hurtado H, Knoops B, van den Bosch de Aguilar P. Rat sciatic nerve regeneration in semipermeable artificial tubes. Exp Neurol 1987;97:751-7.

10. Knoops B, Hurtado H, van den Bosch de Aguilar P. Rat sciatic nerve regeneration within an acrylic semipermeable tube and comparison with a silicon impermeable material. J Neuropathol Exp Neurol 1990; 49:438-48.

11. Jenq $C B$, Coggeshall RE. Nerve regeneration through holey tubes. Brain Res 1985;361:233 41.

12. Descarries L, Schroeder JM. Fixation du tissue nerveux par perfusion a grand débit. J Microscop 1975;7:241-6.

13. Dagnelie P. Théorie et méthodes statistiques. 2nd edition. Gembloux: Les Presses Agronomiques de Gembloux; 1975.

14. Jenq CB, Jenq LL, Coggeshall RE. Nerve regeneration changes with filters of different pore size. Exp Neurol 1987;97:662-71.

15. Jenq $C B$, Coggeshall RE. Permeable tubes increase the length of the gap that regenerating axons can span. Brain Res 1987;408:239-42. 
16. Hurtado $\mathrm{H}$. Peripheral nervous system regeneration in the adult rat: the regeneration chamber (doctoral thesis). Louvain (Belgium): Université Catholique de Louvain; 1990.

17 Kurkinen M, Vaheri A, Roberts PJ, Stenman S. Sequential appearance of fibronectin and collagen in experimental granulation tissue. Lab Invest 1980; $43: 47-51$.

18. Bitterman PB, Rennard SI, Adelberg S, Crystal RC. Role of fibronectin as a growth factor for fibroblasts. J Cell Biol 1983;97:1935-42.

19. Knox P, Crooks S, Rimmer CS. Role of fibronectin in the migration of fibroblasts into plasma clots. J Cell Biol 1986;102:2318-23.

20. Doillon CJ, Silver FH, Berg RA. Fibroblast growth on a porous collagen sponge containing hyaluronic acid and fibronectin. Biomaterials 1987;195-200.

21. Allen-Hoffman LB,Crankshaw CL, Mosher DF. Transforming growth factor $B$ increases cell surface binding and assembly of exogenous (plasma) fibronectin by normal human fibroblasts. Mol Cell Biol 1988;8:234-42.

22. Huang JS, Olsen TJ, Huang SS. The role of growth factors in tissue repair. I.Platelet-derived growth fac- tor. In: Clark RAF, Henson PM, editors. The molecular and cellular biology of wound repair. Plenum Press; 1988:243-53.

23. McCarthy JB, Sas DF, Furcht LT. Mechanisms of parenchymal cell migration into wounds. In: Clark RAF, Henson PM, editors. The molecular and cellular biology of wound repair. Plenum Press; 1988:281-321.

24. Pierce GF, Mustoe TA, Lingelbach J, et al. Plateletderived growth factor and trasforming growth factor $B$ enhance tissue repair activities by unique mechanismes. J Cell Biol 1989;109:429-40.

25. Gabbiani G, Montadon D. Reparative processes in mammalian wound healing: the role of contractile phenomena. Int Rev Cytol 1977;48:187-219.

26. Clark RAF. Overview and general considerations of wound repair. In: Clark RAF, Henson PM, editors. The molecular and cellular biology of wound repair. Plenum Press; 1988:3-34.

27. Williams LR, Varon S. Modification of fibrin matrix formation in situ enhances nerve regeneration in silicone chambers. J Comp Neurol 1985;231:209-20.

28. Liu HM. The role of extracellular matrix in peripheral nerve regeneration: a wound chamber study. Acta Neuropathol 1992;83:469-74. 